\title{
Neurovascular pathophysiology in cerebral ischemia, dementia and the ageing brain - current trends in basic, translational and clinical research
}

\author{
Johannes Boltze ${ }^{1,2^{*}}$, Christoph Kleinschnitz ${ }^{3}$, Klaus G Reymann ${ }^{4,5}$, Georg Reiser ${ }^{6}$, Daniel-Christoph Wagner ${ }^{1,2}$, \\ Alexander $\mathrm{Kranz}^{1,2}$ and Dominik Michalski ${ }^{7}$ for the meeting contributors
}

\begin{abstract}
The $7^{\text {th }}$ International Symposium on Neuroprotection and Neurorepair was held from May $2^{\text {nd }}$ to May $5^{\text {th }}, 2012$ in Potsdam, Germany. The symposium, which directly continues the successful Magdeburg meeting series, attracted over 330 colleagues from 29 countries to discuss recent findings and advances in the field. The focus of the 2012 symposium was widened from stroke and traumatic brain injury to neurodegenerative diseases, notably dementia, and more generally the ageing brain. Thereby, emphasis was given on neurovascular aspects of neurodegeneration and stroke including the blood-brain barrier, recent findings regarding the pathomechanism of Alzheimer's disease, and brain imaging approaches. In addition, neurobiochemical aspects of neuroprotection, the role of astrogliosis, the clinical progress of cell-based approaches as well as translational hurdles and opportunities were discussed in-depth. This review summarizes some of the most stimulating discussions and reports from the meeting.
\end{abstract}

Keywords: Neuroprotection, Neurorepair, Cerebral ischemia, Alzheimer's disease, Small vessel disease, Vascular dementia, Mitochondria, Astrogliosis, in vivo imaging, Translational research

\section{Main text \& conclusions}

Introduction and background information

Vascular disorders of the human brain are a common precondition and a relevant factor of influence for some of the most devastating neurological conditions like stroke and dementia - globally burdening patients, families, and the health care systems. Significant advances have been made regarding our understanding of central pathophysiological aspects of these diseases, as well as possible strategies to counter them. However, we yet have been unable to translate hopeful experimental findings into the clinics to augment the existing, but limited therapeutic options. In the context of ageing industrialized societies with an increasing prevalence of important

\footnotetext{
* Correspondence: johannes.boltze@izi.fraunhofer.de

${ }^{1}$ Fraunhofer-Institute for Cell Therapy and Immunology, Perlickstr. 1, D-04103 Leipzig, Germany

${ }^{2}$ Translational Centre for Regenerative Medicine, University of Leipzig,

Philipp-Rosenthal-Str. 55, D-04103 Leipzig, Germany

Full list of author information is available at the end of the article
}

risk factors like diabetes and arterial hypertension as well as the limited resources for health care worldwide, diseases affecting the cerebral vasculature are one of the most relevant global health problems. Thus, there is an increasing and still unmet need for an even better understanding of stroke and dementia in order to derive novel therapeutic approaches that clearly have the potential to be of benefit for patients.

The $7^{\text {th }}$ International Symposium on Neuroprotection and Neurorepair $\left(7^{\text {th }}\right.$ ISN\&N) was held from May $2^{\text {nd }}$ to May $5^{\text {th }}$ at the Templin Lake in Potsdam, Germany. In continuation of the Magdeburg meeting series and the $6^{\text {th }}$ ISN\&N [1], the meeting was organized jointly by four German institutes, the Fraunhofer Institute of Cell Therapy and Immunology (Leipzig), the Leibniz Institute for Neurobiology, the German Center for Neurodegenerative Diseases (DZNE) of the Helmholtz Society as well as Institute for Neurobiochemistry from the Medical faculty of the Otto-von-Guericke-University (all Magdeburg). A central objective of the $7^{\text {th }}$ ISN\&N was to widen the 
scientific scope of the meeting series from stroke to dementia and more generally the ageing brain, primarily focusing on Alzheimer's disease (AD) and vascular dementia. This paper reviews some of the most interesting findings being presented at the $7^{\text {th }}$ ISN\&N and gives a compact summary of selected scientific debates that emerged during the meeting.

\section{A common burden: vascular aspects of neurodegeneration in stroke and dementia}

Cerebral small vessel diseases are a major cause for chronic cerebral ischemia leading to cognitive decline and behavioral changes, but may also induce stroke-like symptoms if vessels in important brain regions are affected. Holger Braun (Magdeburg, Germany) demonstrated the sequence of events during small vessel alterations in brains of spontaneously hypertensive stroke prone rats. Accumulations of erythrocytes were shown to be the initial step in the pathological cascade, which is followed by blood-brain barrier (BBB) impairments finally leading to microbleeds that cause necrotic tissue damage in terms of microinfarcts [2]. Interestingly, the progress of small vessel alterations simultaneously develops in the kidney, demonstrating that the cerebral microangiopathology is part of a systemic vessel disease in those animals. Clinical research and experimental work suggests that cerebral blood flow is impaired in $\mathrm{AD}$, what may contribute to the detrimental cognitive effects. Nozomi Nishimura (Ithaca, USA) hypothesized that inflammation could play a role in such reductions in blood flow in $\mathrm{AD}$ by leukocyte plugging of capillaries. In vivo two-photon excited fluorescence microscopy was used to examine cortical blood flow in mouse models of AD. To determine whether or not individual brain microvessels are stalled, the movement of nonfluorescent blood cells within the dye-labeled blood plasma was monitored [3]. Thereby, several capillaries were found to plug longer by leucocytes in $\mathrm{AD}$ mice compared to wild type mice. Additional evidence for remodeling of the cerebral microvasculature in $\mathrm{AD}$ comes from magnetic resonance angiography. Didier Leys (Lille, France) provided a review of stroke and dementia as consequences of an altered vasculature, which was followed by a lively round table discussion.

\section{An interesting 'frontier': targeting the blood-brain barrier integrity}

In recent years, the $\mathrm{BBB}$ received increasing attraction in stroke and neurodegeneration, since the concept of a primary separating role (for instance leading to edema and hemorrhage when the BBB integrity is lost) was added by a functional perspective including inflammatory aspects $[4,5]$. Thereby, mediators like cytokines contribute to leukocyte migration, maintaining the inflammatory response with predominantly damaging effects [6]. Despite the uncertainty of the exact cellular composition forming the $\mathrm{BBB}$ along the vascular tree [7], the neurovascular unit (NVU) represents an accepted concept that embeds the BBB into the network of microvessels, glial cells and neurons [4,8]. Gregory J. del Zoppo (Seattle, USA) presented an overview on the barrier structure within the NVU: Since previous research predominantly addressed inter-endothelial cell tight junction complexes, growing evidence indicated that also the adhesion between endothelial cells and the extracellular matrix (arranged amongst others by $\beta 1$-integrin) contribute to BBB permeability [9]. This perspective is supported by the recently demonstrated increase in IgG extravasation after intrastriatal injection of an antibody inhibiting the function of $\beta 1$-integrin in mice [10]. Berislav V. Zlokovic (Los Angeles, USA) stressed the NVU with emphasis on neurodegenerative processes, in particular AD. Thereby, a reduced clearance of $\beta$-amyloid peptides is assumed to emerge from $\mathrm{BBB}$ alterations, contributing to neuronal dysfunction and finally dementia [5]. In this context, pericytes were found to act as key players in $\mathrm{BBB}$ regulation with the potency to promote both BBB breakdown and integrity [11], thus representing a target for future research. While extending this perspective by glial cells, recent research yielded that different isoforms of apolipoprotein E, originating from astrocytes, have opposite effects on the release of matrix metalloproteinase-9 (MMP-9), an enzyme known to mediate BBB breakdown [12]. Dominik Michalski (Leipzig, Germany) focused on side effects of recombinant tissue plasminogen activator (rtPA), currently representing the best available drug-related treatment in acute stroke. Despite the hypothesis that detrimental actions could be attenuated by co-administered hyperbaric oxygen, recent findings failed to support this theoretically forced approach [13]. However, further research is required to develop neuroprotective strategies which can be applied simultaneously with rtPA. Mariel G. Kozberg (New York, USA) introduced new approaches to characterize the NVU by in vivo imaging in rodents. Thereby, optical-based techniques were applied to provide information on blood flow, vessel dilations and rate of oxygenation in different types of vessels and the parenchyma. Two-photon microscopy was found to image neurovascular coupling by using intravenously applied agents for concomitant labeling of cells and vessels. Guido Stoll (Würzburg, Germany) compared different contrast agents for detecting BBB dysfunction in magnetic resonance imaging (MRI), whereas gadofluorine $\mathrm{M}$ was found to best characterize the size of injury after cortical ischemia in mice [14]. Further, the link between BBB disruption and inflammation was established with MRI enhanced by gadolinium-diethylenetriamine penta-acetic acid (DTPA) 
and superparamagnetic iron oxide particles (SPIOs). Thereby, infiltrating macrophages as visualized by SPIOs were found to occur within the area of impaired BBB integrity after cortical ischemia [15], which was not a requested condition in experimental autoimmune encephalomyelitis [16]. These data indicate that the infiltration of inflammatory cells is not compulsorily associated with $\mathrm{BBB}$ opening as indicated by leakage of gadolinium-DTPA or gadofluorine $\mathrm{M}$.

\section{Small molecules with large impact I: new insides into the} role of $A \beta$ in Alzheimer's dementia

While focusing on $\mathrm{AD}$ as a major topic of the $7^{\text {th }}$ ISN\&N, Hans-Ulrich Demuth (Halle, Germany) emphasized that toxicity in $\mathrm{AD}$ is induced by pyroglutamate (PGLU)-A $\beta$, and furthermore, depends on tau protein. Demuth demonstrated that the cytotoxicity is propagated by a prion-like templating mechanism of $A \beta$ misfolding initiated by PGLU-A $\beta$ and that these PGLU-A $\beta$-containing low-molecular weight oligomers remain stable for days in contrast to oligomers not formed by the PGLU-A $\beta$ initiated mechanism [17]. Jens Pahnke (Rostock and Magdeburg, Germany) reported that the BBB transporter $\mathrm{ABCC} 1$ has an important role in cerebral $\mathrm{A} \beta$ clearance and accumulation. Moreover, a pharmacological activation of $\mathrm{ABC}$ transporters can slow down the neurodegenerative cascade [18].

\section{Small molecules with large impact II: underlying mechanisms of neuroprotective approaches in stroke, ageing and neurodegenerative diseases}

Within the numerous efforts to develop novel or even to improve existing neurochemical approaches, topical sessions of the $7^{\text {th }}$ ISN\&N focused on promising molecular targets and on mitochondrial processes. The role of a transcription factor family that is denoted as peroxisome proliferator-activated receptor (PPAR) was presented by several speakers. First, Douglas Feinstein (Chicago, USA) reported on the potential use of PPAR $\gamma$ sparing thiazolidinediones for the treatment of neurodegenerative diseases. These substances, also known as glitazones, are a class of drugs already used in the treatment of diabetes mellitus type 2. Second, Michael Heneka (Bonn, Germany) described PPAR $\gamma$ as a mediator of protection in $\mathrm{AD}$, and third, Anne Murphy (La Jolla, USA) dealt with the value of a preclinical systematic review to analyze mitochondria as targets of thiazolidinediones. In addition, Georg Reiser (Magdeburg, Germany) concentrated on the role of mitochondria to describe a major principle for neuroprotection. His report covered the permeability transition in mitochondria [19] and thereby described a second neuroprotective route: the activated protein $\mathrm{C} /$ endothelial protein $C$ receptor signaling system, which was shown as a promising target in several stroke models. Finally, Raghu Vemuganti (Madison, USA) reported that ubiquitin-like protein modifications are neuroprotective after stroke.

\section{Seeing is believing: novel approaches in imaging the affected brain}

In vivo imaging of structures and processes in living animals as disease models is commonly used to directly prove a stated experimental hypothesis. In the context of continuous technical advances in imaging techniques and analyzing tools, this research field qualifies to solve two major tasks: imaging of direct functional adaptation and changes in plasticity after brain injury, and further, to characterize the morphological structures on microarchitectural levels. During the meeting, the latest studies which focused on the development of MRI, positron emission tomography (PET) and live cell imaging for this purpose were discussed. Alan Koretsky's group (Bethesda, USA) recently used high field MRI to distinguish the arterial and venous hemodynamic response in individual intracortical vessels after whisker pad stimulation [20]. By using high spatial $(150 \mu \mathrm{m})$ and temporal (200 ms) resolution they described the spatio-temporal pattern of the blood oxygenation level dependent (BOLD) fMRI signal and clarified the influence of the micro- and macrovascular architecture. Rick Dijkhuizen (Utrecht, The Netherlands) brought new insides in the reorganization and functional recovery after stroke by comparing graph-based analyses of fMRI signals in gray matter, quantification of fractional anisotropy in white matter by diffusion tensor imaging (DTI) and functional recovery in relation to stroke severity [21]. The group was able to show significant correlations between increased functional connectivity (especially in the area of the sensorimotor cortex) augmented fiber tracts mainly in the ipsilesional corticospinal tract -and better outcome in the sensorimotor performance score. Using contrast-enhanced microangiography, Jan Klohs (Zurich, Switzerland) showed an age-dependent reduction in the number of functional intra-cortical microvessels in aged (24 months) arcA $\beta$ mice. This reduced density of transcortical vessels (with radii less than 20-80 $\mu \mathrm{m}$ ) was attributed to an impaired perfusion and vascular occlusion caused by deposition of $A \beta$ and fibrin [22]. The Florbetaben study, a multicenter phase II study which could identify amyloid- $\beta$-PET as a visual adjunct for the routine diagnostic of dementia, was presented by Henryk Barthel (Leipzig, Germany) representing a translational approach which impressively linked preclinical and clinical research [23]. As an example for clinical stroke research, Marc Fisher (Worcester, USA) summarized clinical trials which used imaging criteria as a decision instrument for the application of recanalization 
therapies. It was shown that especially MRI is useful to identify subgroups which clearly benefit from a consecutive thrombolytic therapy, also at comparatively late stages.

\section{Friend or foe: the immune system in post-stroke pathophysiology}

The interesting role of the immune system in the pathophysiology of cerebral ischemia was highlighted in the session 'Balancing Immune Responses in Neurodegeneration'. Roland Veltkamp (Heidelberg, Germany) emphasized the 'Janus-faced' nature of T cells in stroke development. While several groups could meanwhile confirm that $\mathrm{CD}^{+}$and $\mathrm{CD}^{+} \mathrm{T}$ cells act detrimental during the acute phase of stroke, the contribution of other $\mathrm{T}$ cell subsets, in particular regulatory $\mathrm{T}$ cells (Treg), remains controversial. In line with the description of a beneficial role of Treg for stroke recovery and repair, recently published by his group, Veltkamp now outlined a promising approach to pharmacologically enhance Treg immune functions in stroked mice. In contrast, the group of Christoph Kleinschnitz (Würzburg, Germany) failed to confirm a neuroprotective effect of Treg during the course of cerebral ischemia. By using a mouse model of selective FoxP3 ${ }^{+}$Treg ablation they could even identify Treg as key mediators of ischemic neurodegeneration - at least during the early phase after transient middle cerebral artery occlusion [24]. As potential reasons for these opposed findings different stroke models and immunological tools were lively discussed by the speakers and audience. Thereby, Ulrich Dirnagl (Berlin, Germany) took this controversy as a good example to stress the need for randomized, multicenter preclinical stroke trials. Further emphasis was placed on the role of polymorphonuclear leukocytes and microglia in stroke. Emmanuel Pinteaux (Manchester, UK) pointed out that central and peripheral inflammations represent key drivers of tissue damage in response to acute brain injury such as stroke, and are associated with poorer outcome in stroke patients. He demonstrated that interleukin (IL)-1 is primarily expressed by activated microglia, and acts on astrocytes to release MMP-9, known to contribute on neuronal cell death. IL1 was also found to activate the brain endothelium leading to neutrophil infiltration into the brain and neuronal death, a mechanism that is associated with neutrophil MMP-9 activity and the degradation of the extracellular matrix [25]. Investigating the penumbra by intracranial two-photon microscopy, Jens Neumann (Magdeburg, Germany) revealed an elevated slow rolling of neutrophil granulocytes on the endothelium followed by an infiltration of these cells into the brain. Contradicting data were shown by Britta Engelhardt (Bern, Switzerland), who provided evidence that neutrophil granulocytes do not enter the parenchyma but stay in close proximity to the vessels.

\section{More than just a scar: the Janus face of astrogliosis}

Reactive astrogliosis has long been identified as a simple scarring process in the central nervous system (CNS) that ultimately impairs neural regeneration. However, recent evidence challenges this paradigm, notably by combining genetic ablation of intermediary filaments with different models of CNS diseases. Hence, the scientific community is now reconsidering the unidirectional view on reactive astrocytes and is developing a more differentiated approach. Michael V. Sofroniew (Los Angeles, USA) presented a comprehensive overview on the role of astrocytes in the normal and damaged CNS and particularly emphasized the morphological and functional heterogeneity of reactive astrogliosis. Beyond reactive astrogliosis and its implications, astrocytes might also contribute to CNS disorders either by a loss of normal, or a gain of detrimental functions. Sofroniew concluded that the understanding of these processes might uncover novel therapeutic targets. Magdalena Götz (Munich, Germany) highlighted the potential beneficial action of reactive astrocytes to develop neural stem cell properties beyond the classical CNS niches. These characteristics can be observed after different types of CNS injury and are principally regulated by Sonic hedgehog signaling. In vivo imaging of reactive astrogliosis using two-photon microscopy supported the observation of astrocytic heterogeneity: while many astrocytes remained unchanged after focal CNS damage, a subpopulation of astrocytes showed proliferation within the vascular niche. Milos Pekny (Gothenburg, Sweden) first introduced a novel three-dimensional culture system for astrocytes that largely preserved the complex cellular morphology and reduced the baseline activation which is a common drawback of conventional astrocyte cultures. Pekny further provided evidence for a differentiated role of reactive astrogliosis with beneficial effects in the context of $\mathrm{AD}$ and Batten disease, but a detrimental action during amyotrophic lateral sclerosis. Finally, he proposed that reactive astrogliosis and its consequences are diseasespecific. Additionally, Karsten Ruscher (Lund, Sweden) linked a common treatment of Parkinson's disease with stroke while reporting on the beneficial effects of levodopa on functional recovery after stroke. As a conclusion, he hypothesized that peri-infarct astrocytes equipped with dopamine receptors may contribute to these effects.

\section{Entering the clinics: recent progress in stem cell-based research}

For almost two decades, cell- and stem cell-based approaches are considered among the most promising 
options to treat degenerative diseases of the brain for which regenerative treatments have not been available so far. Even though cell-based therapies have not been proven beneficial in human patients, first data are available from clinical phase I and II trials. In parallel, more concepts emerged to late preclinical phases. Induced pluripotent stem cells (iPSC) were reported to have capacities comparable to those of embryonic stem cells, but can be generated from autologous, patient-specific somatic cells [26]. Brigitte Onteniente (Paris, France) showed that intracerebrally administered human iPSCderived neural progenitor cells differentiate into astrocytes, endothelial cells and GABAergic neurons after transient cerebral ischemia in the rat, ameliorating motor deficits and secondary neurodegeneration. Robert Mays, cofounder and Senior Director of Athersys Inc. (Cleveland, USA) introduced the company's multipotent adult progenitor cell (MAPC) program, which currently includes clinical phase II testing. MAPC were shown to improve functional recovery and to act neuroprotectively by promoting immune system homeostasis following experimental stroke. An open-label, single arm clinical phase I study using intravenous administration of autologous bone marrow mononuclear cells (BM MNC), which also have been thoroughly investigated in preclinical experiments, was conducted by Sean Savitz (Houston, USA) and colleagues [27]. Obtaining $1 \times 10^{7}$ BM MNC per kilogram bodyweight by iliac crest puncture as well as subsequent density gradient centrifugation and reinfusion of the cells within 72 hours following stroke onset were shown both feasible and safe in 10 patients. A clinical phase IIb study is actually being prepared, also assessing efficacy endpoints. Intraparenchymal stem cell administration is considered beneficial even in subacute and chronic stages after cerebral ischemia [28,29]. The Department of Neurosurgery at Stanford University participates in numerous early stage clinical trials for intraparenchymal administration of various stem cell types. Gary Steinberg (Palo Alto, USA) reported encouraging safety results from these trials. In summary, cell-based approaches have tremendously advanced during the last years now being under clinical evaluation. However, it is still unsure whether these approaches can overcome the translational roadblock and show efficacy in human stroke.

\section{Roadblocks, bypasses, and alternative directions: how to move on in translational research?}

Virtually all approaches to transfer experimental therapies for stroke and dementia into the clinics have failed so far, mainly due to a lack of efficacy and/or safety. While the reasons for this dilemma (being referred to as the 'translational roadblock') are still a matter of debate, it appears likely that this roadblock will be hard to overcome [4]. Wolf-Rüdiger Schäbitz (Bielefeld, Germany) reported on the most recent drug candidate that got stuck on its way into the clinic. Granulocyte colony-stimulating factor (G-CSF), although being carefully evaluated in experimental studies [30] and proven safe in a phase IIa trial [31], recently failed to be effective in the phase IIb AXIS 2 study. Schäbitz also drew relevant conclusion of what may be improved to prevent such failures in future studies. Particularly, the patient population heterogeneity needs to be taken into account by testing the influence of age, comorbidities and polypharmacology in preclinical studies, also using large animal models to more closely mimic the human condition. On the other hand, there is no good reason to believe that the translational failure is an inevitable event. Ulrich Dirnagl gave some very interesting examples for relevant results that were 'found in translation', thereby proving evidence that preclinical stroke research can be predictive. For example, the penumbra concept was first reported in primate studies and was reconstructed in rodent models while the time window for intravenous thrombolysis [32] as well as beneficial effects of statins on stroke outcome closely resemble in preclinical and clinical studies. Dirnagl also suggested novel concepts for a more predictable experimental research such as multi-center, international 'phase III' preclinical trials. We still need, however, to find out what particularly prevents so many research activities to be predictive for the human situation.

\section{Conclusions}

The $7^{\text {th }}$ ISN\&N attracted more than 330 scientific colleagues from 29 countries on 6 continents to discuss recent findings and novel concepts in several types of brain injury. Personal comments of numerous attendees, a growing interest in the meeting's topics indicated by steadily increasing numbers of participants as well as very stimulating discussions emerging throughout the meeting underlined the unbroken demand for a forum tailored to discuss our research in the addressed fields. Consequently, the $8^{\text {th }}$ International Symposium on Neuroprotection and Neurorepair will take place from April $9^{\text {th }}$ to April $12^{\text {th }}$, also going back to the roots of the meeting series in Magdeburg, Germany - were the series originally was started in 1998 . The $8^{\text {th }}$ ISN\&N will strengthen the focus on pathophysiological aspects of the altered vasculature in the ischemia-affected and ageing brain.

\section{Abbreviations}

AD: Alzheimer's disease; BBB: Blood-brain barrier; BM MNC: Bone marrow mononuclear cells; BOLD: Blood oxygenation level dependent; CD: Cluster of differentiation; CNS: Central nervous system; DTI: Diffusion tensor imaging; DTPA: Diethylenetriamine penta-acetic acid; DZNE: Deutsches Zentrum für Neurodegenerative Erkrankungen; G-CSF: Granulocyte colony-stimulating factor; IL: Interleukin; iPSC: Induced pluripotent stem cells; 
IgG: Immunoglobulin G; ISN\&N: International Symposium on Neuroprptection \& Neurorepair; MAPC: Multipotent adult progenitor cell; MMP-9: Matrix metalloproteinase-9; MRI/fMRI: (functional) magnetic resonance imaging; NVU: Neurovascular unit; PET: Positron emission tomography; PGLU: Pyroglutamate; PPAR: Peroxisome proliferator-activated receptor; rtPA: Recombinant tissue plasminogen activator; SPIO: Superparamagnetic iron oxide particle; Treg: Regulatory T cell; UK: United Kingdom; USA: United States of America.

\section{Competing interests}

$J B, K R, G R, D C W$ and $A K$ were organizers of the $7^{\text {th }} I S N \& N ; G R, D M$ and $C K$ were invited speakers to the conference. None of the authors has to disclaim any other, particularly commercial or financial competing interests.

\section{Author's contributions}

All authors contributed actively to the conception, writing and revision of the manuscript, and approved its final version. JB coordinated the writing process and was the conference president of the $7^{\text {th }}$ ISN\&N.

\section{Acknowledgements}

The authors want to thank all people and institutions, who actively helped to make the $7^{\text {th }}$ ISN\&N a successful meeting. In particular, we are grateful for generous grants provided by the Deutsche Forschungsgemeinschaft (grant number: RE563/19-1), the Bundesministerium für Bildung und Forschung, BMBF (grant number: 131-101397), the International Brain Research Organisation, the International Society for Neurobiochemstry, and for support and contributions of industrial sponsors. We further want to acknowledge all co-workers who helped to organize the meeting and make it running smoothly, especially the eventlab congress organizing team for their continuous and exceptional support.

\section{Author details}

'Fraunhofer-Institute for Cell Therapy and Immunology, Perlickstr. 1, D-04103 Leipzig, Germany. ${ }^{2}$ Translational Centre for Regenerative Medicine, University of Leipzig, Philipp-Rosenthal-Str. 55, D-04103 Leipzig, Germany. ${ }^{3}$ Department of Neurology, University of Würzburg, Josef-Schneider-Str. 11, D-97080 Würzburg, Germany. ${ }^{4}$ Leibniz Institute for Neurobiology, Project Group Neuropharmacology, Brenneckestr. 6, D-39118 Magdeburg, Germany. ${ }^{5}$ German Centre for Neurodegenerative Diseases (DZNE), site Magdeburg, Leipziger Str. 44, D-39120 Magdeburg, Germany. ${ }^{6}$ Institute of Neurobiochemistry, Otto-von-Guericke-University Magdeburg, Leipziger Str. 44, D-39120 Magdeburg, Germany. ${ }^{7}$ Department of Neurology, University of Leipzig, Liebigstr. 20, D-04103 Leipzig, Germany.

Received: 31 July 2012 Accepted: 3 August 2012

Published: 10 August 2012

\section{References}

1. Boltze J, Kranz A, Wagner DC, Reymann K, Reiser G, Hess DC: Recent advances in basic and translational stroke research. Expert Rev Neurother 2011, 11:199-202.

2. Schreiber S, Bueche CZ, Garz C, Kropf S, Angenstein F, Goldschmidt J, Neumann J, Heinze HJ, Goertler M, Reymann KG, Braun H: The pathologic cascade of cerebrovascular lesions in SHRSP: is erythrocyte accumulation an early phase? J Cereb Blood Flow Metab 2012, 32:278-290.

3. Nguyen J, Nishimura N, Fetcho RN, ladecola C, Schaffer CB: Occlusion of cortical ascending venules causes blood flow decreases, reversals in flow direction, and vessel dilation in upstream capillaries. I Cereb Blood Flow Metab 2011, 31:2243-2254.

4. Endres M, Engelhardt B, Koistinaho J, Lindvall O, Meairs S, Mohr JP, Planas A, Rothwell N, Schwaninger M, Schwab ME, Vivien D, Wieloch T, Dirnagl U: Improving outcome after stroke: overcoming the translational roadblock. Cerebrovasc Dis 2008, 25:268-278.

5. Zlokovic BV: Neurovascular pathways to neurodegeneration in Alzheimer's disease and other disorders. Nat Rev Neurosci 2011, 12:723-738.

6. Kaur C, Ling EA: Blood brain barrier in hypoxic-ischemic conditions. Curr Neurovasc Res 2008, 5:71-81.

7. Bechmann I, Galea I, Perry VH: What is the blood-brain barrier (not)? Trends Immunol 2007, 28:5-11.
8. del Zoppo GJ: The neurovascular unit in the setting of stroke. J Intern Med 2010, 267:156-171.

9. del Zoppo GJ, Milner R: Integrin-matrix interactions in the cerebral microvasculature. Arterioscler Thromb Vasc Biol 2006, 26:1966-1975.

10. Osada T, Gu YH, Kanazawa M, Tsubota Y, Hawkins BT, Spatz M, Milner R, del Zoppo GJ: Interendothelial claudin-5 expression depends on cerebral endothelial cell-matrix adhesion by $\beta(1)$-integrins. J Cereb Blood Flow Metab 2011, 31:1972-1985.

11. Winkler EA, Bell RD, Zlokovic BV: Central nervous system pericytes in health and disease. Nat Neurosci 2011, 14:1398-1405.

12. Bell RD, Winkler EA, Singh I, Sagare AP, Deane R, Wu Z, Holtzman DM, Betsholtz C, Armulik A, Sallstrom J, Berk BC, Zlokovic BV: Apolipoprotein E controls cerebrovascular integrity via cyclophilin A. Nature 2012, 485:512-516.

13. Michalski D, Pelz J, Weise C, Kacza J, Boltze J, Grosche J, Kamprad M, Schneider D, Hobohm C, Härtig W: Early outcome and blood-brain barrier integrity after co-administered thrombolysis and hyperbaric oxygenation in experimental stroke. Exp Trans/ Stroke Med 2011, 3:5.

14. Stoll G, Kleinschnitz C, Meuth SG, Braeuninger S, Ip CW, Wessig C, Nölte I, Bendszus M: Transient widespread blood-brain barrier alterations after cerebral photothrombosis as revealed by gadofluorine M-enhanced magnetic resonance imaging. J Cereb Blood Flow Metab 2009, 29:331-341.

15. Kleinschnitz C, Bendszus M, Frank M, Solymosi L, Toyka KV, Stoll G: In vivo monitoring of macrophage infiltration in experimental ischemic brain lesions by magnetic resonance imaging. I Cereb Blood Flow Metab 2003, 23:1356-1361

16. Stoll G, Bendszus M: Imaging of inflammation in the peripheral and central nervous system by magnetic resonance imaging. Neuroscience 2009, 158:1151-1160.

17. Nussbaum JM, Schilling S, Cynis H, Silva A, Swanson E, Wangsanut $T$, Tayler K, Wiltgen B, Hatami A, Rönicke R, Reymann K, Hutter-Paier B, Alexandru A, Jagla W, Graubner S, Glabe CG, Demuth HU, Bloom GS: Prion-like behaviour and tau-dependent cytotoxicity of pyroglutamylated amyloid- $\beta$. Nature 2012, 485:651-655.

18. Krohn M, Lange C, Hofrichter J, Scheffler K, Stenzel J, Steffen J, Schumacher T, Brüning T, Plath AS, Alfen F, Schmidt A, Winter F, Rateitschak K, Wree A, Gsponer J, Walker LC, Pahnke J: Cerebral amyloid- $\beta$ proteostasis is regulated by the membrane transport protein $A B C C 1$ in mice. J Clin Invest 2011, 121:3924-3931.

19. Azarashvili T, Stricker R, Reiser G: The mitochondria permeability transition pore complex in the brain with interacting proteins - promising targets for protection in neurodegenerative diseases. Biol Chem 2010, 391:619-629.

20. Yu X, Glen D, Wang S, Dodd S, Hirano Y, Saad Z, Reynolds R, Silva AC, Koretsky AP: Direct imaging of macrovascular and microvascular contributions to BOLD fMRI in layers IV-V of the rat whisker-barrel cortex. Neuroimage 2012, 59:1451-1460.

21. van Meer MP, Otte WM, van der Marel K, Nijboer CH, Kavelaars A, van der Sprenkel JW, Viergever MA, Dijkhuizen RM: Extent of bilateral neuronal network reorganization and functional recovery in relation to stroke severity. J Neurosci 2012, 32:4495-4507.

22. Klohs J, Baltes C, Princz-Kranz F, Ratering D, Nitsch RM, Knuesel I, Rudin M: Contrast-enhanced magnetic resonance microangiography reveals remodeling of the cerebral microvasculature in transgenic ArcAbeta mice. J Neurosci 2012, 32:1705-1713.

23. Barthel H, Gertz HJ, Dresel S, Peters O, Bartenstein P, Buerger K, Hiemeyer F, Wittemer-Rump SM, Seibyl J, Reininger C, Sabri O: Cerebral amyloid-beta PET with florbetaben (18 F) in patients with Alzheimer's disease and healthy controls: a multicentre phase 2 diagnostic study. Lancet Neurol 2011, 10:424-435.

24. Kleinschnitz C, Schwab N, Kraft P, Hagedorn I, Dreykluft A, Schwarz T, Austinat M, Nieswandt B, Wiendl H, Stoll G: Early detrimental T-cell effects in experimental cerebral ischemia are neither related to adaptive immunity nor thrombus formation. Blood 2010, 115:3835-3842.

25. Allen C, Thornton P, Denes A, McColl BW, Pierozynski A, Monestier M, Pinteaux E, Rothwell NJ, Allan SM: Neutrophil Cerebrovascular Transmigration Triggers Rapid Neurotoxicity through Release of Proteases Associated with Decondensed DNA. J Immunol 2012, 189:381-392.

26. González F, Boué S, Izpisúa Belmonte JC: Methods for making induced pluripotent stem cells: reprogramming à la carte. Nat Rev Genet 2011, 12:231-242. 
27. Savitz SI, Misra V, Kasam M, Juneja H, Cox CS Jr, Alderman S, Aisiku I, Kar S, Gee A, Grotta JC: Intravenous autologous bone marrow mononuclear cells for ischemic stroke. Ann Neurol 2011, 70:59-69.

28. Smith EJ, Stroemer RP, Gorenkova N, Nakajima M, Crum WR, Tang E, Stevanato L, Sinden JD, Modo M: Implantation site and lesion topology determine efficacy of a human neural stem cell line in a rat model of chronic stroke. Stem Cells 2012, 30:785-796.

29. Shyu WC, Lin SZ, Chiang MF, Su CY, Li H: Intracerebral peripheral blood stem cell $\left(\mathrm{CD} 34^{+}\right)$implantation induces neuroplasticity by enhancing beta1 integrin-mediated angiogenesis in chronic stroke rats. J Neurosci 2006, 26:3444-3453.

30. Philip M, Benatar M, Fisher M, Savitz SI: Methodological quality of animal studies of neuroprotective agents currently in phase II/III acute ischemic stroke trials. Stroke 2009, 40:577-581.

31. Schäbitz WR, Laage R, Vogt G, Koch W, Kollmar R, Schwab S, Schneider D, Hamann GF, Rosenkranz M, Veltkamp R, Fiebach JB, Hacke W, Grotta JC, Fisher M, Schneider A: AXIS: a trial of intravenous granulocyte colonystimulating factor in acute ischemic stroke. Stroke 2010, 11:2545-2551.

32. Zhu H, Fan X, Yu Z, Liu J, Murata Y, Lu J, Zhao S, Hajjar KA, Lo EH, Wang X: Annexin A2 combined with low-dose tPA improves thrombolytic therapy in a rat model of focal embolic stroke. J Cereb Blood Flow Metab 2010, 30:1137-1146.

doi:10.1186/2040-7378-4-14

Cite this article as: Boltze et al: Neurovascular pathophysiology in cerebral ischemia, dementia and the ageing

brain - current trends in basic, translational and clinical research. Experimental \& Translational Stroke Medicine 2012 4:14.

\section{Submit your next manuscript to BioMed Central and take full advantage of:}

- Convenient online submission

- Thorough peer review

- No space constraints or color figure charges

- Immediate publication on acceptance

- Inclusion in PubMed, CAS, Scopus and Google Scholar

- Research which is freely available for redistribution 